\title{
Extradural glomus tumor of the thoracic spine: case report and review of the literature
}

\author{
Xinxing $\operatorname{Li}^{1} \wedge$, Jihui Zheng ${ }^{2} \wedge$ \\ ${ }^{1}$ Department of Neurosurgery, Shengjing Hospital of China Medical University, Shenyang, China; ${ }^{2}$ Department of Radiology, The Fourth Affiliated \\ Hospital of China Medical University, Shenyang, China \\ Correspondence to: Jihui Zheng. Department of Radiology, The Fourth Affiliated Hospital of China Medical University, Shenyang 110004, China. \\ Email: lixx996303@163.com.
}

\begin{abstract}
Glomus tumors are rare lesions that can arise anywhere along the spinal axis. Only 12 cases have been reported. We report a case of a patient with a rare glomangioma of the thoracic spine. Following a review of the 12 previous cases is a discussion of spinal glomus tumors, including clinical presentation, diagnosis, and treatment. A single, recent case arising from the extradural space of the thoracic spine is described, followed by a literature review of spinal glomus tumors. Including our case, 13 cases of glomus tumors have been reported. The mean age at the time of diagnosis was 44.6 years (range, 22-73 years) and the male-to-female (M:F) ratio was 1.17:1. The lesion was located in the cervical spine in 1 patient, the thoracic spine in 7 patients, the lumbar spine in 4 patients, and the sacrum in 1 patient. All the tumors were benign. However, there was 1 report of local recurrence because of incomplete removal. There were no malignant transformations, metastases, or deaths reported, with a mean follow-up of $20.09 \pm 28.43$ months (range, 2-90 months). Spinal glomus tumors are rare, and the preoperative diagnosis is difficult. An overwhelming majority of glomus tumors are benign and are cured by simple local excision. Patients undergoing complete resection have an excellent long-term prognosis.
\end{abstract}

Keywords! Glomangioma; spinal mass; preoperative diagnosis; case report

Submitted Jun 04, 2021. Accepted for publication Jul 13, 2021.

doi: 10.21037/apm-21-1615

View this article at: https://dx.doi.org/10.21037/apm-21-1615

\section{Introduction}

Spinal masses often present challenging diagnostic and therapeutic scenarios. A recent patient with a 5-month history of numbness below his umbilicus, which was determined to be a glomangioma, illustrates this problem. A glomangioma, also known as a glomus tumor, is a benign lesion The incidence of glomus tumor is reportedly $<2 \%$ among all soft tissue tumors. Glomus tumor with spine involvement are extremely rare. In this study, we presented a thoracic glomus tumor with an unusual radiological presentation, which is different from osteolytic intraosseous patterns illustrated before (1-12). This case led us to perform a literature review regarding this rare tumor, which we hoped would enable us to determine the tumor's imaging characteristics and biological behavior, as well as the ability to establish criteria for a preoperative diagnosis. Our analysis of this patient and the current literature are presented. We present the following article in accordance with the CARE reporting checklist (available at https:// dx.doi.org/10.21037/apm-21-1615).

A review of the patient's chart was conducted along with a review of the literature using a Medline search. Translations were performed whenever necessary. Details of the 13 patients (including the current patient) are

\footnotetext{
^ ORCID: Jihui Zheng, 0000-0002-1799-7981; Xinxing Li, 0000-0002-8329-3587.
} 
summarized in Table 1.

\section{Case presentation}

\section{Patient information}

A 47-year-old man presented with a 5-month history of numbness below his umbilicus, especially in his left leg. The patient did not seek treatment until he developed an unsteady gait. Upon neurological examination, the patient had diminished sensation of pinprick and light touch below his umbilicus, and there was weakening of the myodynamics of both lower extremities. Interestingly, his reflexes were symmetric. All procedures performed in studies involving human participants were in accordance with the ethical standards of the institutional and/or national research committee(s) and with the Helsinki Declaration (as revised in 2013). Written informed consent was obtained from the patient.

\section{Clinical findings}

The hematological findings were within normal limits. Magnetic resonance imaging (MRI) showed an extradural lesion at the T7 vertebral body, extending to the T9 vertebral body, with low signal intensity on T1-weighted images (T1WI). In addition, bright enhancement occurred following gadolinium administration (Figure 1). The lesion showed high signal intensity on T2-weighted images (T2WI). The spinal cord was severely compressed, and the T8 neural foramen was enlarged. However, osseous destruction was not observed.

\section{Histopathological examination}

Specimen sections showed a hypercellular neoplasm with sheets of round-to-polygonal epithelioid cells. Individual nuclei were regular, round-to-ovoid, and lacked significant nuclear atypia or necrosis. The background stroma was hyalinized to a myxoid appearance. Scattered, dilated, capillary-sized vascular channels coursed through the lesion (Figure 2). The neoplastic cells were immune-negative for CD117, CK, CgA, Desmin, Factor VIII, LCA, E-cadherin, NSE, Syn, and S-100. Immunoreactivity to SMA, vimentin, CD34, PAS, and CD99 was noted (Figure 3). This staining pattern, in conjunction with the histological features, was consistent with the diagnosis of a glomangioma. This case is considered to be the second case with these characteristics reported in the literature.

\section{Therapeutic intervention}

Surgical intervention was proposed because of the progression of neurological deficits. The patient underwent a T8-T9 hemilaminectomy and complete resection of the extradural tumor. The tumor had an irregular capsule, and slight bleeding occurred when it was incised. After undergoing posterior decompression, his sensory deficit was stable. However, he had regained normal strength in both legs and was living independently.

\section{Follow-up and outcomes}

At his 3-month follow-up examination, the patient stated that he had fully recovered and had no difficulty in walking. He had returned to work and was very satisfied with the results of his surgery.

\section{Results}

A Medline search of the literature found 12 cases of a spinal glomus tumor. The details of these patients and our case are summarized in Table 1.

The mean age at the time of diagnosis was 44.6 years (range, 22-73 years) and the male-to-female (M:F) ratio was $1.17: 1$. The lesion was located in the cervical spine in 1 patient, the thoracic spine in 7 patients, the lumbar spine in 4 patients, and the sacrum in 1 patient. All the patients underwent posterior decompression and 1 patient underwent emergency embolization of the segmental arteries (adhesive and coil embolization) because of profuse bleeding that could not be controlled with other measures. All 13 lesions were located in the extradural space of the spine. The dura was not involved in any case. However, bone structure involvement, erosion, and lytic lesions were found in 8 of the 13 patients.

There were postoperative follow-ups for 11 of the 13 patients from the literature review, as well as our patient. The mean follow-up of the 13 patients was 20.09 \pm 28.43 months (range, 2-90 months). The longest followup was 7.5 years. One case of local recurrence was found in the literature review. This was due to incomplete removal. No malignant transformations, metastases, or deaths 


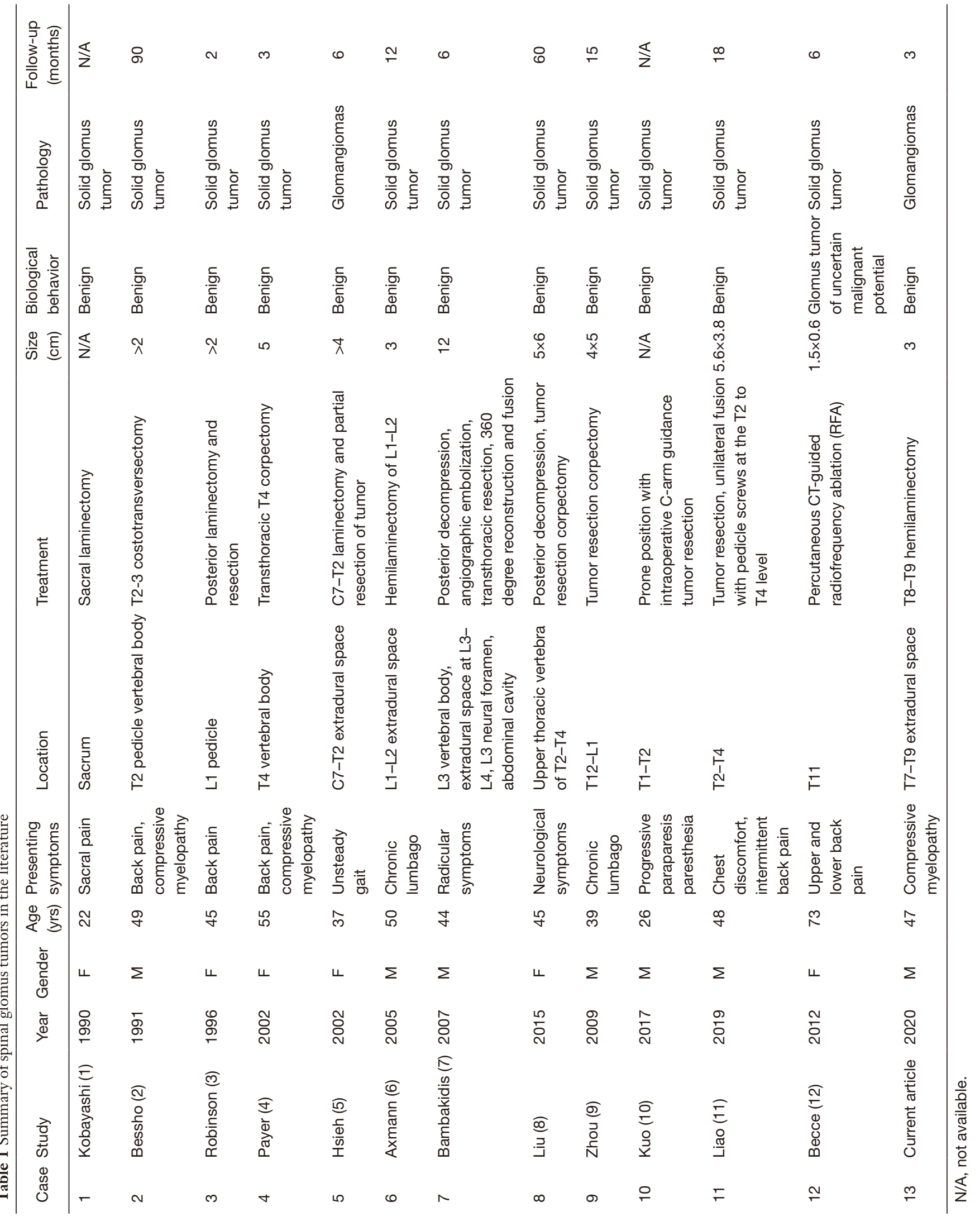



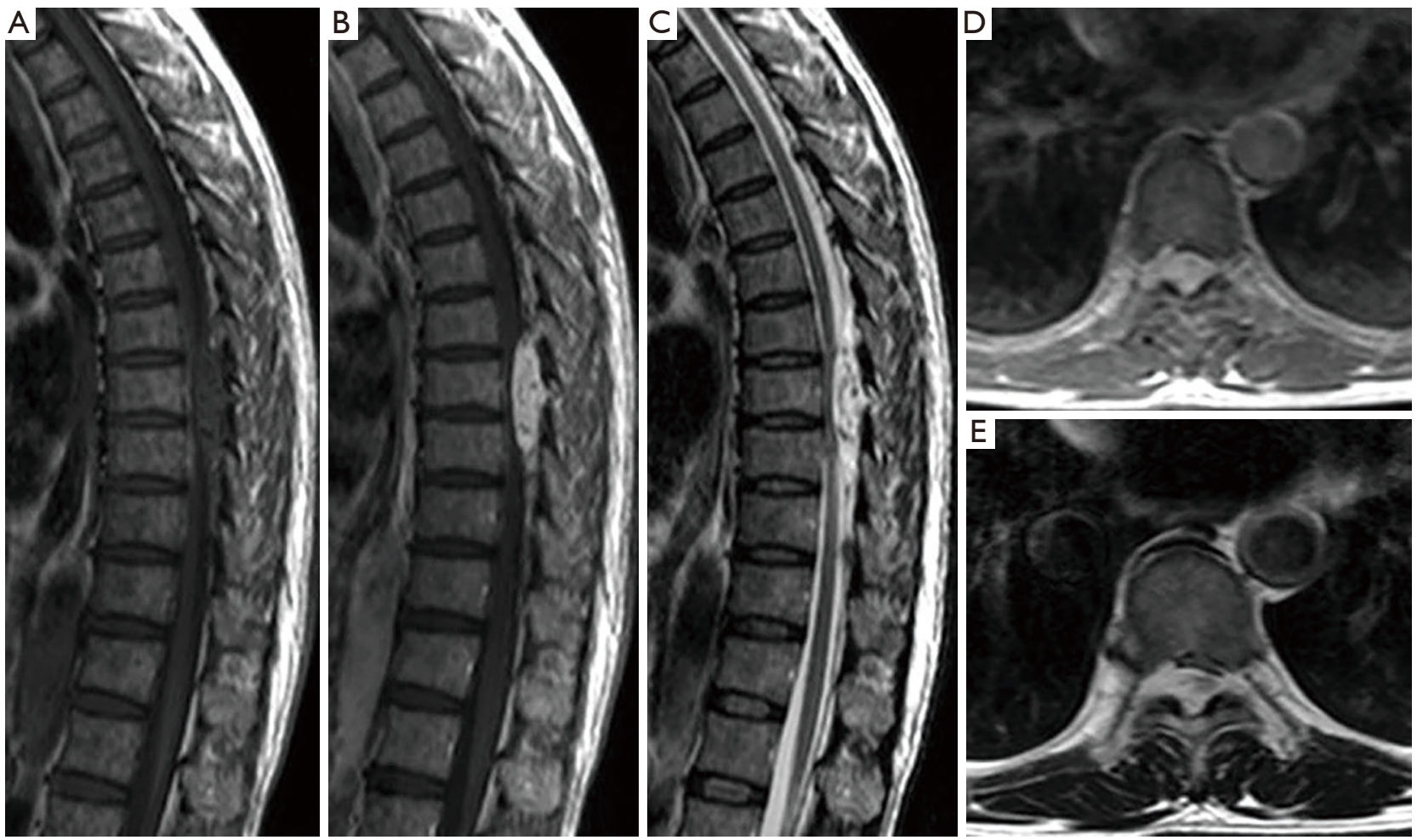

Figure 1 Magnetic resonance imaging showing an extradural lesion in the T7 vertebral body, extending to the T9 vertebral body, compressing the thecal sac. (A) The mass is of low signal intensity on sagittal T1WI; (B) following gadolinium administration, sagittal T1WI showed bright enhancement; (C) abnormally high signal intensity on sagittal T2WI was limited by the tumor envelope. There were numerous signal-void fistular lesions on sagittal T1WI, T2WI, and postcontrast T1WI in the lesion (A-C); (D) enhanced axial T1WI showing that the T8 neural foramen was enlarged; (E) an axial T2WI showing that the T8 neural foramen was enlarged.

occurred at the conclusion of the follow-up periods.

\section{Discussion}

Glomangiomas, a subset of glomus tumors that have a rich vascular network, are neoplasms of the glomus apparatus. The main cause leading to glomus tumor is unknown. In some cases, trauma may be the cause. Glomus tumors are benign neoplasms of the perivasculature. They arise from modified smooth muscle cells called glomus cells, and are located in the walls of the Sucquet-Hoyer canal, which is a specialized arterial-venous anastomosis center that controls blood flow for thermal regulation.

Glomus tumors are most commonly present in the subungual region of the finger. Other common sites include the palm, wrist, forearm, and foot (13-16). In fact, it is now recognized that the tumor also develops in sites where a normal glomus body may be sparse or even absent, such as the patella, long bones, stomach, colon, liver, nerves, eyelids, nose, mediastinum, and mesentery (17-24). A subungual presentation is more common in women. However, no sex predilection is evident at other sites. The male-to-female (M:F) ratio was 1.17:1 in our series. Multiple tumors are more often hereditary and painless, and are more frequent on the arms and have no sex predilection. Multiple lesions may rarely be associated with arteriovenous fistulae, nodular lesions of finger joints, type II multiple endocrine neoplasia, and bone changes such as brachydactyly (25-27).

Glomus tumors are composed of varying proportions of glomus cells, vascular structures, and smooth muscle cells. Depending on the predominant component, there are 3 variants of glomus tumors. When glomus tumors have prominent glomus cells, poor vasculature, and a scant smooth muscle component, they are classified as solid glomus tumors. Glomus tumors with a prominent vascular component are classified as glomangiomas. Finally, when there is a prominent smooth muscle component, the tumors are classified as glomangiomyomas. Solid glomus 

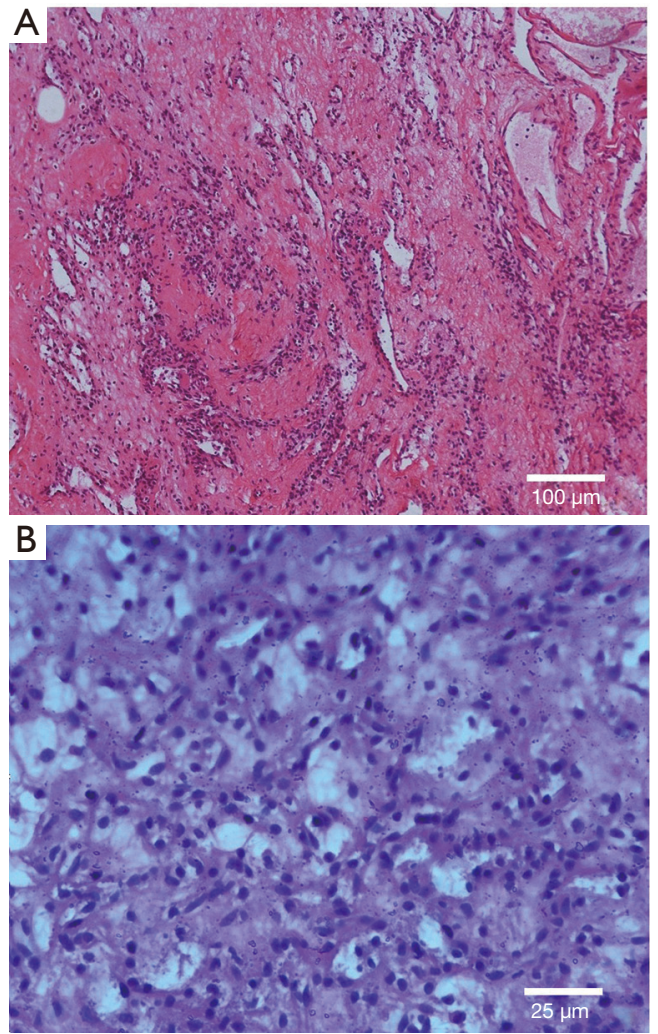

Figure 2 Specimen sections HE staining. (A) The neoplasm is composed of numerous small-to-medium branched vessels surrounded by small round-to-oval uniform cells (H\&E; original magnification $\times 100$ ); (B) the neoplastic cells have sharply outlined round-to-oval nuclei with bland chromatin and scant-to-moderate eosinophilic cytoplasm $(\mathrm{H} \& \mathrm{E}$; original magnification $\times 400)$.

tumors are the most common variant $(75 \%)$, followed by glomangiomas (20\%) and glomangiomyomas (5\%) (28). In our series, 2 of the 13 cases were glomangiomas, while the other 11 were solid glomus tumors (1-12).

Pathologic examination is the gold standard for glomus tumor. Microscopically, glomus tumors present as uniform round-to-ovoid glomus cells arranged as lobules, strands, or broad sheets in a brickwork-like manner. The association with vascular structures includes indistinct capillaries in the walls of the surrounding large blood vessels as well as highly altered vessels. Uniform epithelioid glomus cells with inconspicuous-to-well-defined nucleoli are found between the sinusoidal vessels. Despite their epithelioid appearance and intimate association with the vasculature, glomus cells are immunoreactive with vimentin and SMA. However, a small number of these tumors may co-express myogenic markers and CD34. The current case was immunoreactive with SMA, vimentin, CD34, PAS, and CD99.

Irrespective of their anatomical location, glomus tumors are usually benign. However, rare malignant cases have been described (29,30). Recently, Folpe proposed a classification of atypical and malignant glomus tumors. Malignant glomus tumors are defined as tumors with: (I) large size $(>2 \mathrm{~cm})$ and deep location, (II) atypical mitotic figures, or (III) nuclear atypia. In contrast, glomus tumors of uncertain malignant potential are superficial in location and show high mitotic activity, are large in size, or are deeply located (31). In their study, there were 9 deeply located peripheral soft tissue glomus tumors, and all of them were larger than $2 \mathrm{~cm}$, while only 5 cases metastasized. In a series of 32 gastrointestinal glomus tumors, which by definition were deeply located, only 1 case metastasized, although 19 were larger than $2 \mathrm{~cm}$ (32). In our case review, all of the lesions were $>2 \mathrm{~cm}$ and deeply located. The mean follow-up of the 13 patients together with the current patient was $20.09 \pm 28.43$ months (range, 2-90 months), and the longest follow-up was 7.5 years. No malignancies had appeared at the conclusion of the follow-up periods. Therefore, the risk of metastasis of the deep-seated glomus tumors to the peripheral orgens appears to be markedly different from those located in other deep regions. An extracutaneous, deep location should not be considered a sign of malignancy. On the basis of these criteria and the lack of atypical mitotic figures, our patient's tumor would be classified as benign. Occasionally, glomus tumors occur in a hereditary manner. The gene has been localized to chromosome 1p21-1p22, which codes for glomulin, a protein thought to play a role in vascularization $(33,34)$.

The preoperative diagnosis of spinal glomus tumor is challenging. Usually, plain radiographs are unremarkable for spinal glomus tumors. Most patients with a spinal mass undergo a CT scan and MRI. Although CT images can show well-defined lesions in a few cases, MRI is considered to be an excellent diagnostic modality. On T1WIs, glomus tumors may present as a sharply marginated lesion with hypointensity or isointensity, while on T2WI they are mostly hyperintense. Furthermore, they usually show strong intense enhancement on T1WI after intravenous administration of gadolinium-DTPA (16). Most glomus tumors are surrounded by an envelope. T2WI and T1WI after intravenous administration of contrast medium can detect the envelope as a low signal intensity rim. The envelope, which may be incomplete, may be the consequence of a secondary reaction with the surrounding tissue. An envelope-like structure was observed in our 

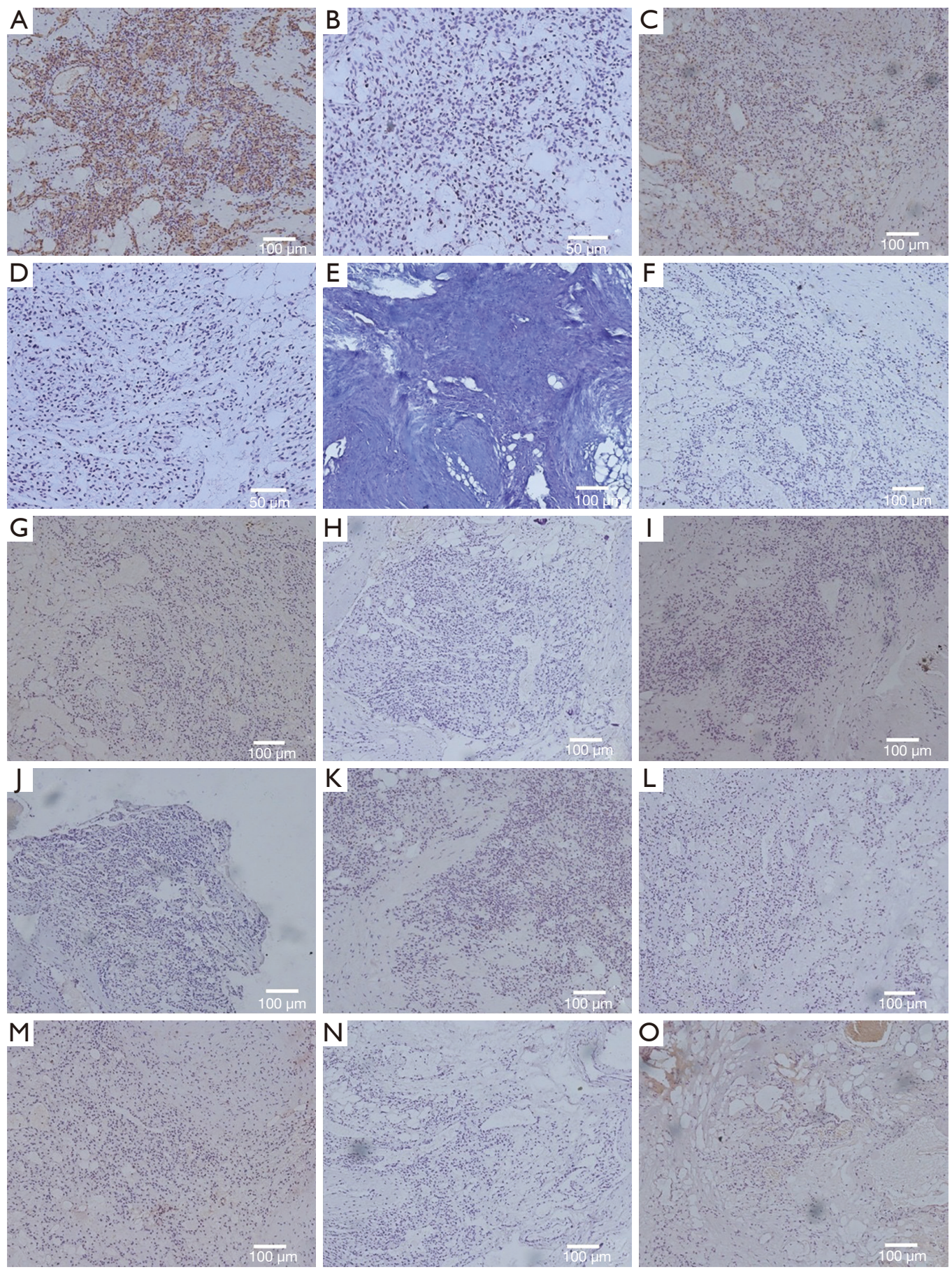

Figure 3 Immunohistochemically, tumor cells stained strongly for CD34, vimentin, and SMA (A-C), and a few cells stained faintly for CD99 and PAS (D,E). Tumor cells were negative for CD117, CK, CgA, Desmin, Factor VIII, LCA, E-cadherin, NSE, Syn, and S-100 (F-O). (A) CD34, original magnification $\times 100$; (B) Vimentin, original magnification $\times 200$; (C) SMA, original magnification $\times 100$; (D) CD99, original magnification $\times 200$; (E) PAS, original magnification $\times 100$; (F) CD117, original magnification $\times 100$; (G) CK, original magnification $\times 100$; (H) CgA, original magnification $\times 100$; (I) Desmin, original magnification $\times 100$; (J) Factor VIII, original magnification $\times 100$; (K) LCA, original magnification $\times 100$; (L) E-cadherin, original magnification $\times 100$; (M) NSE, original magnification $\times 100$; (N) Syn, original magnification $\times 100$; (O) S-100, original magnification $\times 100$. 
case. The MR images of our case are fairly similar to the MR features of the other cases. However, a characteristic finding in our case was the signal-void fistular lesion in the tumor. The features of the fistular lesion were similar to the images of vascular structures. It was also comparable to the histological findings of tissue with a prominent vascular component. The fistular lesion, due to the direction of flow, was identified on the sagittal images but not on the axial images.

In our case, the differential diagnosis included primary extradural masses or intradural extramedullary lesions of the spine. Schwannomas, which are the most common extradural tumors, show the typical dumbbell-shaped appearance (extra- and intradural portions) in $50 \%$ of cases. However, $50 \%$ are completely extradural. Schwannomas on MRI images tend to have low signal intensity on T1WI and have brightly increased signal intensity on T2WI due to the high-water content of these lesions. These lesions are usually markedly enhanced following gadolinium administration. The MR findings of the 8 cases showed they had similar MR features to schwannomas, except for the signal-void fistular lesion. Ganglioneuromas and neuroblastomas are tumors of childhood, which often appear with large paraspinal tumor masses.

To date, the most effective treatment for glomus tumor is surgical resection. The choice of therapeutic strategy is predicated on the lesion location and clinical presentation. An overwhelming majority of glomus tumors are benign and are cured by simple local excision. The postsurgical recurrence rate is approximately $10 \%$ because of incomplete removal. In the literature we reviewed, with the longest follow-up being 7.5 years, there was 1 report of local recurrence due to incomplete removal. However, no malignant transformations, metastases, or deaths occurred by the conclusion of the follow-up period.

\section{Conclusions}

In summary, spinal glomus tumor, although rare, deserves consideration during the differential diagnosis of spinal lesions. While preoperative diagnosis is difficult, certain imaging features can provide more information to base the diagnosis on, and raise the level of certainty. Differentiation of glomus tumors from schwannomas can be accomplished on the basis of histology and immunostaining. An overwhelming majority of glomus tumors are benign and are cured by simple local excision.

\section{Acknowledgments}

Funding: This work is supported by grants from the Liaoning Science and Technology Plan Project (No. 2018225094) and the Natural Science Foundation of Liaoning Province (No. 2020-MS-165).

\section{Footnote}

Reporting Checklist: The authors have completed the CARE reporting checklist. Available at https://dx.doi. org/10.21037/apm-21-1615

Conflicts of Interest: Both authors have completed the ICMJE uniform disclosure form (available at https://dx.doi. org/10.21037/apm-21-1615). The authors have no conflicts of interest to declare.

Ethical Statement: The authors are accountable for all aspects of the work in ensuring that questions related to the accuracy or integrity of any part of the work are appropriately investigated and resolved. All procedures performed in studies involving human participants were in accordance with the ethical standards of the institutional and/or national research committee(s) and with the Helsinki Declaration (as revised in 2013). Written informed consent was obtained from the patient.

Open Access Statement: This is an Open Access article distributed in accordance with the Creative Commons Attribution-NonCommercial-NoDerivs 4.0 International License (CC BY-NC-ND 4.0), which permits the noncommercial replication and distribution of the article with the strict proviso that no changes or edits are made and the original work is properly cited (including links to both the formal publication through the relevant DOI and the license). See: https://creativecommons.org/licenses/by-nc-nd/4.0/.

\section{References}

1. Kobayashi Y, Kawaguchi T, Imoto K, et al. Intraosseous glomus tumor in the sacrum. A case report. Acta Pathol Jpn 1990;40:856-9.

2. Bessho $\mathrm{Y}$, Kataoka $\mathrm{O}$, Sho $\mathrm{T}$, et al. Intraosseous glomus tumor in the upper thoracic spine complicating compression myelopathy. A case report. Spine (Phila $\mathrm{Pa}$ 1976) 1991;16:988-90. 
3. Robinson JC, Kilpatrick SE, Kelly DL Jr, et al. Intraosseous glomus tumor of the spine. Case report and review of the literature. J Neurosurg 1996;85:344-7.

4. Payer M, Grob D, Benini A, et al. Intraosseous glomus tumor of the thoracic spine. Case illustration. J Neurosurg 2002;96:137.

5. Hsieh TJ, Kuo YT, Tsai KB, et al. Glomangioma at the cervical spine as an extradural dumbbell-shaped tumor--a case report. Kaohsiung J Med Sci 2002;18:253-6.

6. Axmann C, Feiden W, Moeller V, et al. Paravertebral glomus tumors. Skeletal Radiol 2005;34:112-5.

7. Bambakidis NC, Gore P, Eschbacher J, et al. Intraosseous spinal glomus tumors: case report. Neurosurgery 2007;60:E1152-3; discussion E1153.

8. Liu T, Zou W, Kong J, et al. Embolization in the treatment of an intraosseous glomus tumor in the upper thoracic spine complicating compression myelopathy: a case report and a literature review. Turk Neurosurg 2015;25:479-84.

9. Zhou P, Zhang H, Bu H, et al. Paravertebral glomangiomatosis. Case report. J Neurosurg 2009;111:272-7.

10. Kuo CH, Huang WC, Wu JC, et al. Unusual imaging presentation of spinal glomus tumor: case report. J Spine Surg 2017;3:715-8.

11. Liao Z, Chen C, Wang B, et al. Minimally invasive resection of a glomus tumor of the thoracic spine: a case report and literature review. J Int Med Res 2019;47:2746-53.

12. Becce F, Richarme D, Letovanec I, et al. Percutaneous radiofrequency ablation of primary intraosseous spinal glomus tumor. Skeletal Radiol 2012;41:467-72.

13. Santoshi JA, Kori VK, Khurana U, et al. Glomus tumor of the fingertips: A frequently missed diagnosis. J Family Med Prim Care 2019;8:904-8.

14. Çevik HB, Çiçek ÇA, Kayahan S, et al. Glomus tumors of the upper extremity. Handchir Mikrochir Plast Chir 2021;53:72-5.

15. Morey VM, Garg B, Kotwal PP, et al. Glomus tumours of the hand: Review of literature. J Clin Orthop Trauma 2016;7:286-91.

16. Trehan SK, Soukup DS, Mintz DN, et al. Glomus Tumors in the Foot: Case Series. Foot Ankle Spec 2015;8:460-5.

17. Li L, Xu QX, Zhang XY, et al. Unusual location of the glomus tumour in the liver: A case report and literature review. Medicine (Baltimore) 2018;97:e11294.

18. El Hyaoui H, Messoudi A, Rafai M, et al. Unusual localization of glomus tumor of the knee. Joint Bone Spine 2016;83:213-5.
19. Namikawa T, Tsuda S, Fujisawa K, et al. Glomus tumor of the stomach treated by laparoscopic distal gastrectomy: A case report. Oncol Lett 2019;17:514-7.

20. Chen IY, Fazili BG, Liao X, et al. Glomus Tumor of the Colon: A Rare Case Report and Review of Literature. Int J Surg Pathol 2020;28:691-5.

21. Saxe SJ, Grossniklaus HE, Wojno TH, et al. Glomus cell tumor of the eyelid. Ophthalmology 1993;100:139-43.

22. de Bruin AF, Verhoef C, den Bakker MA, et al. Glomus tumor of the mesentery with atypical features: a case report. Int J Surg Pathol 2008;16:440-2.

23. Kanakis M, Rapti N, Chorti M, et al. Asymptomatic Glomus Tumor of the Mediastinum. Case Rep Surg 2015;2015:631625

24. Meguro S, Kusama Y, Matsushima S, et al. Nasal glomus tumor: A rare nasal tumor with diffuse and strongly positive synaptophysin expression. Pathol Int 2019;69:672-4.

25. Liszka H, Panikowski R, Kwolek K, et al. Multiple Glomus Tumors in the Left Foot of 41-year-old Woman. A Case Report. Ortop Traumatol Rehabil 2020;22:195-201.

26. Ceccato GHW, Rassi MS, Borba LAB, et al. Microsurgical Resection of Multiple Giant Glomus Tumors. J Neurol Surg B Skull Base 2019;80:S385-8.

27. Kiyosawa T, Umebayashi Y, Nakayama Y, et al. Hereditary multiple glomus tumors involving the glans penis. A case report and review of the literature. Dermatol Surg 1995;21:895-9.

28. Ariizumi Y, Koizumi H, Hoshikawa M, et al. A primary pulmonary glomus tumor: a case report and review of the literature. Case Rep Pathol 2012;2012:782304.

29. Chen JH, Lin L, Liu KL, et al. Malignant glomus tumor of the intestinal ileum with multiorgan metastases: A case report and review of literature. World J Gastroenterol 2020;26:770-6.

30. Braham E, Zairi S, Mlika M, et al. Malignant glomus tumor of trachea: a case report with literature review. Asian Cardiovasc Thorac Ann 2016;24:104-6.

31. Folpe AL, Fanburg-Smith JC, Miettinen M, et al. Atypical and malignant glomus tumors: analysis of 52 cases, with a proposal for the reclassification of glomus tumors. Am J Surg Pathol 2001;25:1-12.

32. Miettinen M, Paal E, Lasota J, et al. Gastrointestinal glomus tumors: a clinicopathologic, immunohistochemical, and molecular genetic study of 32 cases. Am J Surg Pathol 2002;26:301-11.

33. Boon LM, Brouillard P, Irrthum A, et al. A gene for inherited cutaneous venous anomalies ("glomangiomas") 
localizes to chromosome 1p21-22. Am J Hum Genet 1999;65:125-33.

34. Brouillard P, Boon LM, Mulliken JB, et al. Mutations in a novel factor, glomulin, are responsible for glomuvenous malformations ("glomangiomas"). Am J Hum Genet 2002;70:866-74.

(English Language Editor: C. Betlazar-Maseh)

Cite this article as: Li X, Zheng J. Extradural glomus tumor of the thoracic spine: case report and review of the literature. Ann Palliat Med 2021;10(8):9309-9317. doi: 10.21037/apm-21-1615 